\title{
NONLINEAR WAVES AND SHOCK CALCULATIONS FOR HYPERELASTIC FLUID-FILLED TUBES*
}

\author{
BY \\ T. BRYANT MOODIE AND GORDON E. SWATERS \\ L'niversity' of Alberta, Edmonton, Canada
}

\begin{abstract}
The propagation of both finite amplitude and weakly nonlinear waves in fluid-filled hyperelastic tethered tubes subjected to axial strain is investigated in some detail. Procedures based upon the methods of characteristics and relatively undistorted waves are deployed to compute the time and location of first shock formation in tubes having both constant and variable properties ahead of the wave. The influence of wall thickness changes in shock formation is explored and it is further found that if the transmural pressure ahead of the wave is zero then no shock can form on the lead characteristic for any model based on a rational theory of finite elasticity. This latter result is in disagreement with several earlier studies.
\end{abstract}

1. Introduction. In this paper we investigate the nonlinear propagation of pressure pulses along thin walled, tethered, hyperelastic, and axially strained tubes containing homogeneous, incompressible, and inviscid fluids. It is possible, in the model developed here, to incorporate a compressible fluid in the analysis, but if the fluid is a liquid with, say, the density and compressibility of water it is felt that this effect will be negligible. Also, we might include the effect of fluid viscosity, but in a number of practical applications, say for parameters appropriate to biological problems involving the larger blood vessels, this effect is also negligible [1].

We develop a one-dimensional model dealing with variations of the various quantities involved in the axial direction only. The radial variations are not completely neglected, however, as shown in [2], and the equations used result from a perturbation of the two-dimensional axially symmetric equations. Under the assumption that all disturbances have a long wavelength, the relationship between the transmural pressure $p_{t r}^{*}$, the local cross sectional area $A^{*}$, and the axial coordinate $x^{*}$ may be taken in the form [3]

$$
\Phi\left(A^{*}, p_{t r}^{*}, x^{*}\right)=0,
$$

where $\Phi$ is a functional determined by the elastic properties and initial geometry of the tube. The outside pressure is assumed to be constant, but if this is not so only a small modification to the theory to be presented is required.

${ }^{*}$ Received January 4, 1989. 
Early investigators (see Womersley [4] and the references in Skalak [5]) used an expression obtained from linear elasticity theory for $\Phi$. Even as recently as 1979, Chen and McCarthy [7] employed the linear theory of elasticity in their computations of shock initiation times for fluid-filled tubes. Anliker et al. [7] avoided direct use of finite deformation elasticity by expressing the wave speed $c^{*}$ as a particular function of $p_{t r}^{*}$ and $x^{*}$. Moodie and Haddow [3] first introduced the two-dimensional strain energy function to account for nonlinear elastic deformation of the tube wall in a consistent fashion. Cowley, in a pair of recent papers [8], [9], again deployed the two-dimensional strain energy function to study elastic jumps on fluid-filled tubes and their associated dispersive wave trains. Swaters [18] has developed a theory describing resonantly interacting wave triads in hyperelastic fluid-filled tubes. Tait and Moodie [10], following [3], studied wave propagation and shock formation in nonlinearly elastic and viscoelastic fluid-filled tubes and were able to exhibit an exact solution for a tube composed of a Mooney-Rivlin material.

In this paper we employ a model for fluid-filled elastic tubes which is based upon the theory of finite elastic deformations. These tubes are thin walled and tethered by axial forces that prevent all axial motion [8]. We employ both the method of characteristics, which is an exact method, and the theory of relatively undistorted waves ([11], [12]), a systematic perturbation expansion procedure, to study initial boundary value problems and compute the time and location of first shock formation. The latter approach may be used to yield results for tubes with variable properties when shocks form either on or back of the front. This is in contrast to the acceleration wave analysis of [6] which is capable of predicting shock formation only when such shocks form upon the lead characteristic. Results obtained by the two methods are compared for a particular choice of strain energy function and shown to be in excellent agreement. The method of relatively undistorted waves is them employed to examine the effect that changes in wall thickness have upon the development of shocks. Both methods are capable of yielding the result that for arbitrary strain energy functions and any amount of axial strain, waves will not first break on the lead characteristic when the transmural pressure ahead of the wave is zero.

2. Equations. The nonlinear dimensional governing equations describing the fluid dynamics are the conservation of mass equation given by

$$
\frac{\partial A^{*}}{\partial t^{*}}+\frac{\partial\left(A^{*} u^{*}\right)}{\partial x^{*}}=0
$$

where $A^{*}, x^{*}$ are as defined previously, $t^{*}$ is time, and $u^{*}$ is the fluid velocity in the axial direction, and the momentum equation given by

$$
\frac{\partial u^{*}}{\partial t^{*}}+u^{*} \frac{\partial u^{*}}{\partial x^{*}}+\frac{1}{\rho^{*}} \frac{\partial p_{t r}^{*}}{\partial x^{*}}=0
$$

where $\rho^{*}$ is the constant fluid density. In addition, we require a relationship between $A^{*}, p_{t r}^{*}$, and $x^{*}$ appropriate to the nonlinear elastic model for the tube wall.

We consider axisymmetric deformations of an incompressible hyperelastic fluidfilled tube and assume that the inertia of the wall is always sufficiently small to allow the elasticity to be based upon a quasi-steady theory. Cowley [8], [9], has shown that 
this approximation is valid even if the fluid within the tube is unsteady, provided

$$
\frac{\rho_{u} h_{0}^{*}}{\rho^{*} a_{0}^{*}} \ll 1
$$

where $\rho_{w}, a_{0}^{*}$, and $h_{0}^{*}$ are the wall membrane density, and undeformed wall radius and thickness, respectively.

Since the wall material is incompressible, it can be described by a strain energy function ([3], [13])

$$
W^{*}=W^{*}\left(I_{1}, I_{2}, x^{*}\right)
$$

of the first and second invariants $I_{1}$ and $I_{2}$ of the tensor, $\mathbf{F F}^{T}$ (or $\mathbf{F}^{T} \mathbf{F}$ ), where $\mathbf{F}$ is the deformation gradient tensor and the superscript $T$ denotes the transpose. The variable $x^{*}$ appearing in (2.3) allows for the elastic properties to vary from point to point along the tube. The invariants $I_{1}$ and $I_{2}$, when expressed in terms of the principal stretches $\lambda_{1}$ and $\lambda_{2}$, are given by

$$
\begin{aligned}
& I_{1}=\lambda_{1}^{2}+\lambda_{2}^{2}+\frac{1}{\lambda_{1}^{2} \lambda_{2}^{2}}, \\
& I_{2}=\lambda_{1}^{2} \lambda_{2}^{2}+\frac{1}{\lambda_{1}^{2}}+\frac{1}{\lambda_{2}^{2}} .
\end{aligned}
$$

Since $\lambda_{1}, \lambda_{2}$ represent principal stretches in the azimuthal and longitudinal directions, respectively, we have for a tube subjected to a uniform axial strain $e$ that

$$
\lambda_{1}=\frac{a^{*}}{a_{0}^{*}}, \quad \lambda_{2}=(1+e)\left(1+\left(\frac{\partial a^{*}}{\partial x^{*}}\right)^{2}\right)^{1 / 2},
$$

where $a^{*}$ is the local deformed (i.e., time dependent) tube radius.

From the general theory of thin walled cylindrical hyperelastic shells we have

$$
p_{t r}^{*}=\frac{h_{0}^{*}}{a^{*}(1+e)} \frac{\partial W^{*}}{\partial \lambda_{1}}-\frac{1}{a^{*}} \frac{\partial}{\partial x^{*}}\left[\frac{a_{0}^{*}\left(\partial a^{*} / \partial x^{*}\right) h_{0}^{*}}{\left(1+\left(\partial a^{*} / \partial x^{*}\right)^{2}\right)^{1 / 2}} \frac{\partial W^{*}}{\partial \lambda_{2}}\right] .
$$

Versions of this formula have been presented in [3], [8], [10], and [18]. Combining (2.3)-(2.6) and invoking the long wavelength approximation we arrive at

$$
p_{t r}^{*}=\frac{2 h_{0}^{*}}{a_{0}^{*}(1+e)}\left[\frac{1}{(1+e)^{2}} \frac{\partial W^{*}}{\partial I_{1}}+\frac{\partial W^{*}}{\partial I_{2}}\right]\left[(1+e)^{2}-\left(\frac{A_{0}^{*}}{A^{*}}\right)^{2}\right],
$$

where

$$
\begin{aligned}
& I_{1}=\frac{A^{*}}{A_{0}^{*}}+\frac{A_{0}^{*}}{(1+e)^{2} A^{*}}+(1+e)^{2}, \\
& I_{2}=\frac{A_{0}^{*}}{A^{*}}+(1+e)^{2} \frac{A^{*}}{A_{0}^{*}}+\frac{1}{(1+e)^{2}},
\end{aligned}
$$

and

$$
A^{*}=\pi\left(a^{*}\right)^{2}, \quad A_{0}^{*}=\pi\left(a_{0}^{*}\right)^{2} .
$$

We observe that at zero transmural pressure we must have $A^{*}=A_{0}^{*} /(1+e)$. Note that there is an accompanying area reduction in the presence of a nonzero axial strain. 
It is important to add that $\Phi\left(A^{*}, p_{t r}^{*}, x^{*}\right)$ in (1.1) cannot be completely arbitrary but must take the form imposed by (2.7) if the mechanical properties of the tube wall are to be based upon a rational theory of finite elasticity. In addition, the wellknown mathematical and physical restrictions (e.g., [13]) on strain-energy functions for isotropic Green elastic materials must hold. These requirements will further restrict the allowed functional forms for $\Phi\left(A^{*}, p_{t r}^{*}, x^{*}\right)$.

It will be convenient for further analysis to introduce nondimensional (unasterisked) variables. To this end we specify

$$
\begin{array}{cc}
p_{t r}^{*}\left(x^{*}, t^{*}\right)=\rho^{*}\left(c_{0}^{*}\right)^{2} p(x, t), & u^{*}\left(x^{*}, t^{*}\right)=c_{0}^{*} u(x, t), \\
A^{*}\left(x^{*}, t^{*}\right)=\frac{\bar{A}_{0}^{*}}{1+e} A(x, t), & A_{0}^{*}\left(x^{*}\right)=\bar{A}_{0}^{*} A_{0}(x), \\
a_{0}^{*}\left(x^{*}\right)=\bar{a}_{0}^{*} a_{0}(x), \quad W^{*}\left(I_{1}, I_{2}, x^{*}\right)=W_{*} W\left(I_{1}, I_{2}, x\right), \\
x^{*}=\bar{a}_{0}^{*} x, \quad t^{*}=\frac{\bar{a}_{0}^{*} t}{c_{0}^{*}}, & h_{0}^{*}\left(x^{*}\right)=\bar{h}_{0}^{*} h_{0}(x),
\end{array}
$$

where

$$
\begin{gathered}
\left(c_{0}^{*}\right)^{2}=2 \bar{h}_{0}^{*} W_{*}(1+e), \\
W_{*}=\left.\left[\frac{1}{(1+e)^{2}} \frac{\partial W^{*}}{\partial I_{1}}+\frac{\partial W^{*}}{\partial I_{2}}\right]\right|_{p_{t r}^{*}=0, x^{*}=0}
\end{gathered}
$$

and

$$
\bar{h}_{0}^{*}=\left.h_{0}^{*}\right|_{x^{*}=0}, \quad \bar{a}_{0}^{*}=\left.a_{0}^{*}\right|_{x^{*}=0}, \quad \bar{A}_{0}^{*}=\pi\left(\bar{a}_{0}^{*}\right)^{2} .
$$

The nondimensional equations can be written in the form

$$
\begin{gathered}
\frac{\partial A}{\partial t}+\frac{\partial(A u)}{\partial x}=0, \\
\frac{\partial u}{\partial t}+u \frac{\partial u}{\partial x}+\frac{\partial p}{\partial x}=0, \\
p=\left[1-\left(\frac{A_{0}}{A}\right)^{2}\right]\left[\frac{1}{(1+e)^{2}} \frac{\partial W}{\partial I_{1}}+\frac{\partial W}{\partial I_{2}}\right] \frac{h_{0}(x)}{a_{0}(x)}, \\
I_{1}=\frac{A}{(1+e) A_{0}}+\frac{A_{0}}{(1+e) A}+(1+e)^{2}, \\
I_{2}=(1+e) \frac{A_{0}}{A}+(1+e) \frac{A}{A_{0}}+\frac{1}{(1+e)^{2}} .
\end{gathered}
$$

With the introduction of the dimensional pulse wave velocity $c^{*}$ given by

$$
\left(c^{*}\right)^{2}=\frac{A^{*}}{\rho^{*}}\left(\frac{\partial A^{*}}{\partial p_{t r}^{*}}\right)^{-1}
$$

or, in nondimensional variables, by

$$
c^{2}=A\left(\frac{\partial A}{\partial p}\right)^{-1}
$$

we may rewrite $(2.14)$ in the form

$$
\frac{\partial p}{\partial t}+u \frac{\partial p}{\partial x}+c^{2} \frac{u}{\partial x}+\frac{c^{2}}{A} \frac{\partial A}{\partial x} u=0
$$


The pair of equations (2.15) and (2.21) constitutes a totally hyperbolic system having characteristic directions in the $(x, t)$ plane given by

$$
\frac{d x}{d t}=u \pm c \quad \text { for } C_{ \pm} \text {characteristics }
$$

and the corresponding independent characteristic forms

$$
\frac{d u}{d t} \pm \frac{1}{c} \frac{d p}{d t}+\frac{c}{a} \frac{\partial A}{\partial x} u=0 \quad \text { on } C_{ \pm} .
$$

3. Wave breaking and characteristics. In this section we consider $A, h$, and $a$ to be constant for constant $p$.

3.1. General considerations. Under the above condition the governing equations reduce to

Introducing the hodograph transformation

$$
\left.\begin{array}{r}
\frac{\partial u}{\partial t}+u \frac{\partial u}{\partial x}+\frac{\partial p}{\partial x}=0 . \\
\frac{\partial p}{\partial t}+u \frac{\partial p}{\partial x}+c^{2} \frac{\partial u}{\partial x}=0 .
\end{array}\right\}
$$

$$
(x, t) \mapsto(u, p),
$$

with $x_{u} t_{p}-t_{u} \neq 0$, transforms the system (3.1) into the linear hyperbolic system

$$
\left[\begin{array}{l}
x \\
t
\end{array}\right]_{u}+\left[\begin{array}{cc}
u & c^{2}-u^{2} \\
1 & -u
\end{array}\right]\left[\begin{array}{l}
x \\
t
\end{array}\right]_{p}=0
$$

The eigenvalues of the coefficient matrix in (3.3) are $\pm c$ so that the characteristics in the $(u, p)$ plane are given by

$$
\frac{d p}{d u}= \pm c(p)
$$

Thus

$$
\int \frac{d p}{c(p)} \pm u=\text { constant } \equiv \Gamma_{ \pm}
$$

and these are the Riemann invariants associated with the $C_{ \pm}$characteristics, that is, $\Gamma_{ \pm}$are constant on $C_{ \pm}$, respectively.

We shall consider the initial boundary value problem prescribed by

$$
\begin{gathered}
p=0, \quad u=0, \quad t<0, \quad x>0, \\
\left.p(x, t)\right|_{x=0}=p_{0}(t), \quad t>0 .
\end{gathered}
$$

The lead characteristic divides the $(x, t)$ plane into two regions labelled I ahead (i.e., to the right) of this characteristic wherein $p=u=0$, and II behind it. Since Region II is a $\Gamma_{-}$simple wave [14], $\Gamma_{-}$and $\Gamma_{+}$are both constant on the $C_{+}$characteristics there implying that these must be straight lines.

Since $\Gamma_{-}$is constant on the $C_{-}$characteristics and since $p=u=0$ in Region I, we have

$$
\Gamma_{-}=\int_{0}^{p} \frac{d \tilde{p}}{c(\tilde{p})}-u=0
$$


We note that for the constant state $p=p_{1}, u=u_{1}$ ahead of the pulse the lower limit of integration in (3.7) would be $p_{1}$ and $u$ would be replaced by $u-u_{1}$. In Region II

$$
u=\int_{0}^{p} \frac{d \tilde{p}}{c(\tilde{p})}
$$

and for the $C_{+}$characteristics we have

$$
\frac{d x}{d t}=u+c=\int_{0}^{p} \frac{d \tilde{p}}{c(\tilde{p})}+c(p) \equiv f(p) .
$$

Combining (3.6) and (3.9) then enables us to determine the characteristic through any point $(x, t)$ of Region II. Knowing the pressure at this point then enables us to find $u(x, t)$ from $(3.8)$ and the area from the pressure/area relation.

The equation of the characteristic that intersects $x=0$ at $t=\xi$ is

$$
x=(t-\xi) F(\xi), \quad F(\xi)=f\left(p_{0}(\xi)\right) .
$$

The condition that two (neighbouring) characteristics $\xi$ and $\xi+\delta \xi$ intersect at a point $(x, t)$ is that $(3.10)$ and

$$
x=(t-(\xi+\delta \xi)) F(\xi+\delta \xi)
$$

hold simultaneously. In the limit $\delta \xi \rightarrow 0$, these give

$$
\begin{gathered}
x=(t-\xi) F(\xi), \\
(t-\xi) F^{\prime}(\xi)=F(\xi),
\end{gathered}
$$

for the implicit equations on an envelope. Together these equations show that an envelope is formed in $t>0$ by those characteristics for which

$$
\chi(\xi)=\xi+\frac{F(\xi)}{F^{\prime}(\xi)}>0 .
$$

The minimum value of $t$ on the envelope occurs for that value of $\xi$ for which the $\chi(\xi)$ in (3.12) is a minimum. If we denote this value of $\xi$ by $\xi_{B}$ then the wave first breaks at time $t_{B}$, where

$$
t_{B}=\xi_{B}+\frac{F\left(\xi_{B}\right)}{F^{\prime}\left(\xi_{B}\right)}
$$

and the breaking distance $x_{B}$ is given by

$$
x_{B}=\frac{\left(F\left(\xi_{B}\right)\right)^{2}}{F^{\prime}\left(\xi_{B}\right)},
$$

where $\xi_{B}$ is that root of

$$
2\left(F^{\prime}(\xi)\right)^{2}-F(\xi) F^{\prime \prime}(\xi)=0
$$

producing the global minimum for $\chi(\xi)$.

In Sec. 4 we will analyze the propagation of weakly nonlinear waves using the method of relatively undistorted waves. For these calculations we will assume a boundary condition in which $p_{0}(t)$ in (3.6) has the form

$$
p_{0}(t)=\varepsilon^{1 / 2} g\left(\frac{t}{\varepsilon}\right)
$$


where it will be assumed that $0<\varepsilon \ll 1$. The exact results of this subsection will be used to test the validity of the asymptotic expansion.

3.2. Front breaking waves. Before proceeding to analyze the weakly nonlinear waves we examine the possibility of waves breaking on the lead characteristic. These waves are initiated in axially strained tubes by the action of a general boundary condition of the form (3.6). We make no restriction on the strain energy function describing the tube's elastic properties apart from the usual regularity assumption that it be continuously differentiable [13].

For a wave to break on the front is tantamount to $\xi_{B}=0$ in (3.13) or a breaking time $t_{B}$ given by

$$
t_{B}=\left\{\frac{f\left(p_{0}(\xi)\right) c\left(p_{0}(\xi)\right)}{\left[1+c\left(p_{0}(\xi)\right) c^{\prime}\left(p_{0}(\xi)\right)\right] p_{0}^{\prime}(\xi)}\right\}_{\xi=0} .
$$

From (3.17) we see that a necessary condition for breaking to occur on the front is

$$
\left.\frac{d p_{0}}{d t}\right|_{t=0} \neq 0
$$

Then with $p_{0}$ satisfying (3.18) we see that the breaking time $t_{B}$ cannot be finite unless

$$
\left.c\left(p_{0}(\xi)\right) c^{\prime}\left(p_{0}(\xi)\right)\right|_{\xi=0} \neq-1
$$

This provides a second necessary condition for waves to break on the lead characteristic. The necessary condition (3.19) is equivalent to

$$
3 \frac{d p}{d A}+\left.A \frac{d^{2} p}{d A^{2}}\right|_{A=A_{0}} \neq 0
$$

where we recall that $A=A_{0}$ if and only if $p=0$.

From Eq. (2.16)-(2.18) we have that

$$
\begin{aligned}
\frac{d p}{d A}= & \frac{2 h_{0} A_{0}^{2}}{a_{0} A^{3}}\left[\frac{1}{(1+e)^{2}} W_{1}+W_{2}\right] \\
& +\frac{h_{0}}{a_{0}}\left[1-\left(\frac{A_{0}}{A}\right)^{2}\right]\left[\frac{1}{(1+e)^{2}} W_{11} I_{1}^{\prime}+\frac{1}{(1+e)^{2}} W_{12} I_{2}^{\prime}+W_{12} I_{1}^{\prime}+W_{22} I_{2}^{\prime}\right], \\
\frac{d^{2} p}{d A^{2}}= & \frac{-6 h_{0} A_{0}^{2}}{a_{0} A^{4}}\left[\frac{1}{(1+e)^{2}} W_{1}+W_{2}\right] \\
& +\frac{4 h_{0} A_{0}^{2}}{a_{0} A^{3}}\left[\frac{1}{(1+e)^{2}} W_{11} I_{1}^{\prime}+\frac{1}{(1+e)^{2}} W_{12} I_{2}^{\prime}+W_{12} I_{1}^{\prime}+W_{22} I_{2}^{\prime}\right] \\
& +\frac{h_{0}}{a_{0}}\left[1-\left(\frac{A_{0}}{A}\right)^{2}\right]\left[\frac{1}{(1+e)^{2}} W_{111}\left(I_{1}^{\prime}\right)^{2}+\frac{2}{(1+e)^{2}} W_{112} I_{1}^{\prime} I_{2}^{\prime}\right. \\
& +\frac{1}{(1+e)^{2}} W_{11} I_{1}^{\prime \prime}+\frac{1}{(1+e)^{2}} W_{122}\left(I_{2}^{\prime}\right)^{2} \\
& +\frac{1}{(1+d)^{2}} W_{12} I_{2}^{\prime \prime}+W_{112}\left(I_{1}^{\prime}\right)^{2}+2 W_{122} I_{1}^{\prime} I_{2}^{\prime}
\end{aligned}
$$


where

$$
W_{i_{1} \cdots i_{n}}=\partial^{(n)} W / \partial I_{i_{1}} \cdots \partial I_{i_{n}} .
$$

Direct computation of (3.21) and (3.22) with the forms for $I_{1}$ and $I_{2}$ given in (2.17) and (2.18) implies that when $p=0$ (i.e., $A=A_{0}$ ) ahead of the wave the necessary condition (3.19) or (3.20) for breaking on the front is always violated. Waves which propagate into a region where transmural pressure is zero may never break on the front.

In the next section we develop an asymptotic theory for computing shock initiation times and positions for pulses propagating in fluid-filled tubes whose properties may vary with position. We know from the previous general discussion that such shocks can only form behind the front. Indeed, subsequent numerical studies of the exact solution confirm this finding.

\section{Small-but-finite amplutide pulses in inhomogeneous tubes.}

4.1. Problem formulation. When the mechanical or geometrical properties of the tube wall (or fluid) vary spatially ahead of the propagating pulse the existence of Riemann invariants is precluded and the method of characteristics as developed in Sec. 3 cannot be applied. Indeed, there are very few general analytical procedures that can be used on inhomogeneous wave problems of the type to be considered here [12]. One method yielding both qualitatively and quantitatively accurate predictions for the time and location of first shock formation is the so-called method of relatively undistorted waves ([11], [12]) which is a small-amplitude nonlinear geometrical optics perturbation procedure which systematically generalizes 'Whitham's rule' ([15], [16]) for the nonlinear amplitude correction to the phase in the linear geometrical optics solution. From the nonlinear phase correction it will be possible to determine the time and location of initial shock formation.

We take as the starting point in the analysis the nondimensional problem given by (2.14)-(2.18) together with the boundary condition (3.16) under the assumption $0<$ $\varepsilon \ll 1$. Physically, the parameter $\varepsilon \equiv \bar{a}_{0}^{*}\left(c_{0}^{*} \tau^{*}\right)^{-1}$ where $\bar{a}_{0}^{*}$ and $c_{0}^{*}$ are given by $(2.10)-$ (2.13), and $\tau^{*}$ is an appropriate time scale representing the duration in time of the boundary data. Consequently, $\varepsilon^{-1}$ is a measure of the nondimensional frequency of the boundary pulse relative to the dynamical frequency associated with the equations of motion. Clearly $\varepsilon \rightarrow 0^{+}$will correspond to high-frequency boundary data. It is assumed that $g(t / \varepsilon) \sim O(1)$ in (3.16). It follows, therefore, that $g^{*} \sim \varepsilon^{1 / 2} \rho^{*}\left(c_{0}^{*}\right)^{2}$ where $g^{*}$ is the dimensional scaling for the amplitude of the boundary pulse data and that (3.16) will correspond to a small-amplitude, high-frequency boundary condition under the assumption $0<\varepsilon \ll 1$.

It is important to note that the scaling for (3.16) implies that the order of the nondimensional period of the boundary condition (3.16) (i.e., the period is $O(\varepsilon)$ ) is an order of magnitude smaller (with respect to the expansion parameter $\varepsilon^{1 / 2}$ ) than the amplitude. This scaling will imply that the amplitude correction to the linear geometrical optics phase is a second-order effect. We will see that in the inhomogeneous $O\left(\varepsilon^{1 / 2}\right)$ equations in the perturbation expansion yet-to-be-derived, the required compatibility condition on the inhomogeneity is satisfied for all solutions 
to the $O(1)$ problem under the relatively undistorted wave ansatz. Thus the nonlinear effects in the phase take place at second order.

The small-amplitude scaling will necessitate computing a Taylor expansion for (2.16) of the form (we chose to consider $A=A(p, x)$ )

$$
A=A_{0}(x)+\varphi_{0}(x) p+\varphi_{1}(x) p^{2}+\varphi_{2}(x) p^{3}+O\left(p^{4}\right),
$$

where

$$
\varphi_{n}(x)=\frac{1}{(n+1) !} \frac{\partial^{(n+1)} A}{\partial p^{n+1}} \quad(p=0, x), n=0,1, \ldots
$$

Tedious but straightforward calculation of the $\varphi_{n}$ 's yields

$$
\begin{aligned}
\varphi_{0} & =A_{0}^{3 / 2}(x) /\left[2 h_{0}(x)\left(W_{1}^{0} /(1+e)^{2}+W_{2}^{0}\right)\right], \\
\varphi_{1} & =3 \varphi_{0}^{2}(x) / 2 A_{0}(x), \\
\varphi_{2} & =[(5 / 2)-\beta(x)] \varphi_{0}^{3}(x) / A_{0}^{2}(x),
\end{aligned}
$$

where

$$
\beta(x) \equiv\left[W_{11}^{0} /(1+e)+2(1+e) W_{12}^{0}+(1+e)^{3} W_{22}^{0}\right] /\left[W_{1}^{0}+(1+e)^{2} W_{2}^{0}\right],
$$

where we have introduced the notation

$$
W_{i_{1} \cdots i_{n}}^{0}=\left.\frac{\partial^{(n)} W}{\partial I_{i_{1}} \cdots \partial I_{i_{n}}}\right|_{A=A_{0}} .
$$

The boundary condition (3.16) suggests that the appropriate form of the solution will be given by

$$
\begin{aligned}
& p(x, t)=\varepsilon^{1 / 2} \hat{p}(x, \theta), \\
& u(x, t)=\varepsilon^{1 / 2} \hat{u}(x, \theta),
\end{aligned}
$$

where $\theta$ is the characteristic coordinate given implicitly by the arrival time formula

$$
t=T(x, \theta) \text {. }
$$

The arrival time $T(x, \theta)$ will depend on the solutions $\hat{p}(x, \theta)$ and $\hat{u}(x, \theta)$ and has to be determined as part of the overall solution. With no loss of generality we may impose the natural boundary condition

$$
T(0, \theta)=\varepsilon \theta \text {. }
$$

Note that the relationship between $\theta$ and the characteristic parameter $\xi$ of Sec. 3 is given by $\xi=\varepsilon \theta$.

It follows from (4.5) that for the change of variables $(x, t) \mapsto(x, \theta)$ derivatives will map according to

$$
\begin{gathered}
\partial_{t} \mapsto\left(T_{\theta}\right)^{-1} \partial_{\theta}, \\
\partial_{x} \mapsto \partial_{x}-\left(T_{x} / T_{\theta}\right) \partial_{\theta} .
\end{gathered}
$$

Substitution of (4.1), (4.4), and (4.7) into the original nondimensional problem consisting of $(2.14)-(2.18)$ yields

$$
\begin{aligned}
\varphi_{0} p_{\theta}-T_{x} A_{0} u_{\theta}= & -T_{\theta}\left(A_{0} u\right)_{x}-2 \varepsilon^{1 / 2} \varphi_{1} p p_{\theta}+\varepsilon^{1 / 2} T_{x} \varphi_{0}(p u)_{\theta}-\varepsilon^{1 / 2} T_{\theta}\left(\varphi_{0} p u\right)_{x} \\
& +\varepsilon T_{x} \varphi_{1}\left(p^{2} u\right)_{\theta}-\varepsilon T_{\theta}\left(\varphi_{1} p^{2} u\right)_{x}-3 \varepsilon \varphi_{2} p^{2} p_{\theta}+O\left(\varepsilon^{3 / 2}\right),
\end{aligned}
$$




$$
u_{\theta}-T_{x} p_{\theta}=-T_{\theta} p_{x}+\varepsilon^{1 / 2} T_{x} u u_{\theta}-\varepsilon^{1 / 2} T_{\theta} u u_{x},
$$

where we have dropped the carets from $p(x, \theta)$ and $u(x, \theta)$. The boundary conditions on the solutions to $(4.8)$ can be written as

$$
\begin{gathered}
\left.p(x, \theta)\right|_{x=0}=g(\theta), \\
\left.T(x, \theta)\right|_{x=0}=\varepsilon \theta .
\end{gathered}
$$

4.2. The asymptotic expansion. For the limit $\varepsilon \rightarrow 0^{+}$an asymptotic solution for (4.8) and (4.9) can be found in the form

$$
(p, u, T)=\sum_{n=0}^{\infty} \varepsilon^{n / 2}\left(p^{(n)}, u^{(n)}, T^{(n)}\right)
$$

with corresponding boundary conditions

$$
\begin{gathered}
\left.p^{(n)}(x, \theta)\right|_{x=0}=g(\theta) \delta_{n 0}, \\
\left.T^{(n)}(x, \theta)\right|_{x=0}=\theta \delta_{n 2},
\end{gathered}
$$

for $n \geq 0$, where $\delta_{n m}$ is the usual Kronecker delta function between $n$ and $m$.

Substitution of the asymptotic expansion (4.10) into the governing equations (4.8) yields the homogeneous $O(1)$ problem

$$
\begin{gathered}
\varphi_{0} p_{\theta}^{(0)}-T_{x}^{(0)} A_{0} u_{\theta}^{(0)}=0, \\
T_{x}^{(0)} p_{\theta}^{(0)}-u_{\theta}^{(0)}=0 .
\end{gathered}
$$

For a nontrivial solution it follows that

$$
\begin{gathered}
T_{x}^{(0)}= \pm\left(\varphi_{0} / A_{0}\right)^{1 / 2}, \\
u^{(0)}=T_{x}^{(0)} p^{(0)},
\end{gathered}
$$

where it has been assumed that $u^{(0)}(x, \theta)$ and $p^{(0)}(x, \theta)$ have compact support with respect to $\theta$. We restrict attention to pulses travelling to the right by taking the positive sign in (4.13a). Also, associated with the positive eigenvalue $\left(A_{0} / \varphi_{0}\right)^{1 / 2}$ of the system of equations (4.12) is a left eigenvector $\mathbf{L}=\left(T_{x}^{(0)}, \varphi_{0}\right)$.

The inhomogeneous $O\left(\varepsilon^{1 / 2}\right)$ problem is given by

$$
\begin{aligned}
& \varphi_{0} p_{\theta}^{(1)}-T_{x}^{(0)} A_{0} u_{\theta}^{(1)}= T_{x}^{(1)} A_{0} u_{\theta}^{(0)}-T_{\theta}^{(1)}\left(A_{0} u^{(0)}\right)_{x} \\
&-2 \varphi_{1} p^{(0)} p_{\theta}^{(0)}+T_{x}^{(0)} \varphi_{0}\left(p^{(0)} u^{(0)}\right)_{\theta}, \\
& u_{\theta}^{(1)}-T_{x}^{(0)} p_{\theta}^{(1)}=T_{x}^{(1)} p_{\theta}^{(0)}-T_{\theta}^{(1)} p_{x}^{(0)}+T_{x}^{(0)} u^{(0)} u_{\theta}^{(0)} .
\end{aligned}
$$

Forming the inner product $L \cdot[(4.14 a),(4.15 b)]$ yields the compatibility condition on the $O(1)$ solutions given by

$$
2 \varphi_{0} T_{x}^{(1)} p_{\theta}^{(0)}-T_{\theta}^{(1)}\left[2 \varphi_{0} p_{x}^{(0)}+T_{x}^{(0)}\left(A_{0} T_{x}^{(0)}\right)_{x} p^{(0)}\right]=0
$$

where the relationship $\varphi_{1}=3 \varphi_{0}^{2} /\left(2 A_{0}\right)=(3 / 2) \varphi_{0}\left(T_{x}^{(0)}\right)^{2}$ has been used.

Note that there is no quadratic nonlinear term with respect to $p^{(0)}$ in $(4.15)$. This has occurred because of the relationship between $\varphi_{0}(x)$ and $\varphi_{1}(x)$ as given by (4.3b). 
It is important to realize that this relationship will hold independently of the particular strain energy function assumed for the tube wall. Further, it is easy to show that the relationship (4.3b) is equivalent to the condition derived in Sec. 3 (under a small amplitude limit) for a shock not to form on the lead characteristic (i.e., (3.19) with an equals sign replacing the not equals). Finally, we note that this relationship between $\varphi_{0}(x)$ and $\varphi_{1}(x)$ will also imply that the nonlinear amplitude corrections to the phase are determined from the $O(\varepsilon)$ equations.

The $O\left(\varepsilon^{1 / 2}\right)$ compatibility condition (4.15) contains two unknown functions, i.e., $T^{(1)}(x, \theta)$ and $p^{(0)}(x, \theta)$. In order to be able to make further progress analytically the relatively undistorted wave ansatz is made; namely that for each compatibility condition in the asymptotic hierarchy it is assumed that $O\left(\partial_{\theta} p^{(n)} / p^{(n)}\right) \gg \partial_{x}$. Physically, this local condition expresses the notion that the cross-characteristic gradients vary rapidly in comparison to those along the characteristics which vary relatively slowly (i.e., a high-frequency pulse). Consequently, it follows from (4.15) that the coefficient functions must individually satisfy the conditions

$$
\begin{gathered}
2 \varphi_{0} T_{x}^{(1)}=0, \\
2 \varphi_{0} p_{x}^{(0)}+T_{x}^{(0)}\left(A_{0} T_{x}^{(0)}\right)_{x} p^{(0)}=0 .
\end{gathered}
$$

It follows immediately from $(4.16 \mathrm{a})$ and $(4.11 \mathrm{~b})$ that

$$
T^{(1)}(x, \theta) \equiv 0 \text {. }
$$

Then, from (4.16b), (4.13a), and (4.11a) it follows that

$$
p^{(0)}(x, \theta)=g(\theta)\left[2 \varphi_{0}(x) A_{0}(x)\right]^{-1 / 4},
$$

where the fact that $A_{0}(0)=1$ and $\varphi_{0}(0)=\frac{1}{2}$ has been used (see the scaling definitions (2.10)-(2.13)). The solution (4.18) is, of course, simply the linear geometrical optics solution for $p^{(0)}(x, \theta)$. It follows from (4.17), the second relation in (4.13), and (4.14b) that

$$
u^{(1)}(x, \theta)=T_{x}^{(0)}(x, \theta) p^{(1)}(x, \theta)+\frac{1}{2}\left(T_{x}^{(0)}(x, \theta)\right)^{3}\left(p^{(0)}(x, \theta)\right)^{2},
$$

where again we have assumed that $u^{(1)}(x, \theta)$ and $p^{(1)}(x, \theta)$ have compact support with respect to the phase variable $\theta$.

The equations (4.13) and (4.18) determine the form of the explicit dependency of the $O(1)$ solutions on the 'fast' phase variable $\theta$ and 'slow' space variable $x$. The compatibility condition on the $O(\varepsilon)$ will determine the explicit dependency or the phase variable $\theta$ on The $O(1)$ solutions, and thereby give the small-amplitude correction to the characteristic curves.

The $O(\varepsilon)$ problem can be put into the form

$$
\begin{aligned}
& \varphi_{0} p_{\theta}^{(2)}-T_{x}^{(0)} A_{0} u_{\theta}^{(2)}= T_{x}^{(2)} A_{0} u_{\theta}^{(0)}-T_{\theta}^{(2)}\left(A_{0} u^{(0)}\right)_{x} \\
&-2 \varphi_{1}\left(p^{(0)} p^{(1)}\right)_{\theta}+T_{x}^{(0)} \varphi_{0}\left(p^{(0)} u^{(1)}+u^{(0)} p^{(1)}\right)_{\theta} \\
&+T_{x}^{(0)} \varphi_{1}\left(\left(p^{(0)}\right)^{2} u^{(0)}\right)_{\theta}-3 \varphi_{2}\left(p^{(0)}\right)^{2} p_{\theta}^{(0)} \\
& u_{\theta}^{(2)}-T_{x}^{(0)} p_{\theta}^{(2)}=T_{x}^{(2)} p_{\theta}^{(0)}-T_{\theta}^{(2)} p_{x}^{(0)}+T_{x}^{(0)}\left(u^{(0)} u^{(1)}\right)_{\theta} .
\end{aligned}
$$


Application of the compatibility constraint $\mathbf{L} \cdot[(4.20 \mathrm{a}),(4.20 \mathrm{~b})]$ leads to

$$
p_{\theta}^{(0)}\left\{2 \varphi_{0} T_{x}^{(2)}+\left[3 \varphi_{0}\left(T_{x}^{(0)}\right)^{5}+3 \varphi_{1}\left(T_{x}^{(0)}\right)^{3}-3 \varphi_{2} T_{x}^{(0)}\right]\left(p^{(0)}\right)^{2}\right\}=0 .
$$

Note that there are no terms proportional to $p^{(0)} p^{(1)}, p_{x}^{(0)}$ or $p^{(0)}$. The $p^{(0)} p^{(1)}$ terms vanish because the relationship $\varphi_{1}=(3 / 2)\left(T_{x}^{(0)}\right)^{2} \varphi_{0}$ annihilates the coefficient of this term. The terms proportional to $p_{x}^{(0)}$ and $P^{(0)}$ vanish because of the linear geometrical optics solution (4.18).

If the expressions (4.3b) and (4.3c) are substituted for $\varphi_{1}(x)$ and $\varphi_{2}(x)$ in (4.21) it follows that (because we implicitly assume $p_{\theta}^{(0)} \neq 0$ )

$$
T^{(2)}(x, \theta)=\theta-\frac{3}{2} \int_{0}^{x} \beta(\eta)\left[T_{x}^{(0)}(\eta, \theta)\right]^{5}\left[p^{(0)}(\eta, \theta)\right]^{2} d \eta,
$$

where the boundary condition (4.11a) has been used. Thus, the leading order amplitude corrected formula for the arrival time can be put into the form

$$
\begin{aligned}
t= & \int_{0}^{x}\left[\varphi_{0}(\eta) / A_{0}(\eta)\right]^{1 / 2} d \eta \\
& +\varepsilon\left\{\theta-\left(3 / 2^{3 / 2}\right)(g(\theta))^{2} \int_{0}^{x} \beta(\eta)\left[\varphi_{0}(\eta)\right]^{2}\left[A_{0}(\eta)\right]^{2}\left[A_{0}(\eta)\right]^{-3} d \eta\right\}+O\left(\varepsilon^{3 / 2}\right),
\end{aligned}
$$

which completes the leading order solution. We remark here that the leading order perturbation solution given by (4.18) and (4.23) is formally valid only in a neighbourhood of the lead characteristic $\theta=0$. Consequently, the leading order solution just obtained is not necessarily a global asymptotic representation of the travelling wave pulse.

A simple leading order formula for the location and time of first shock formation can be obtained from (4.23). Since a shock first occurs the first time that $t_{\theta}=0$, it follows that the position of this shock (denoted $x_{B}$ ) will be determined from

$$
\int_{0}^{x_{B}} \beta(\eta)\left[\varphi_{0}(\eta)\right]^{2}\left[A_{0}(\eta)\right]^{-3} d \eta=\frac{\sqrt{2}}{3}\left[g_{\theta}\left(\theta_{B}\right) g\left(\theta_{B}\right)\right]^{-1},
$$

where $\theta=\theta_{B}$ is the maximizer of $g_{\theta}(\theta) g(\theta)$. Once $x_{B}$ is determined from (4.24) the time of initial shock formation, $t_{B}$, will be determined from (4.23) where the righthand side is evaluated with $x=x_{B}$ and $\theta=\theta_{B}$. In Subsection 5.1 we will compare the predictions of our asymptotic theory, assuming a homogeneous tube wall, with the results obtained from the exact theory developed in Sec. 3. In Subsection 5.2 an example calculation with varying tube wall thickness will be presented.

\section{Applications of the asymptotic theory.}

5.1. An example calculation with a homogeneous tube wall. In order to compare the predictions of the weakly nonlinear asymptotic theory developed in Sec. 4 with the exact finite amplitude theory of Sec. 3, we shall compute the location and time of first shock formation for a simple example. We take as the pressure boundary condition

$$
g(\theta)=\alpha \theta\left(1+\theta^{2}\right) \exp \left(-\theta^{2}\right),
$$

where $\alpha$ is a nondimensional amplitude parameter. 
The strain energy function we take is the Ishihara et. al [17] model given by

$$
W\left(I_{1}, I_{2}\right)=b\left(I_{1}-3\right)+(1-b)\left(I_{2}-3\right)+\gamma\left(I_{1}-3\right)^{2},
$$

where $b$ and $\gamma$ are real constants. The strain energy model (5.2) has been proposed as a realistic yet simple model for many elastomers.

It follows from (4.3a) and (4.3d) that

$$
\begin{gathered}
\varphi_{0} \equiv \frac{1}{2}, \\
\beta \equiv 2 \gamma,
\end{gathered}
$$

where $A_{0} \equiv 1.0$ (because of homogeneity), and $e=0.0$ is assumed for convenience. From (4.24) we therefore find that the location of the first shock will be given by

$$
x_{B}=\frac{2^{3 / 2}}{3}\left[\gamma g_{\theta}\left(\theta_{B}\right) g\left(\theta_{B}\right)\right]^{-1},
$$

and that the corresponding time of the first shock will be given by

$$
t_{B}=x_{B} / \sqrt{2}+\varepsilon\left[\theta_{B}-3 \gamma\left(g\left(\theta_{B}\right)\right)^{2} x_{B}(4 \sqrt{2})^{-1}\right],
$$

where $\theta_{B}$ is the maximizer of $g_{\theta}(\theta) g(\theta)$. For the boundary condition (5.1) we find that $\theta_{B} \simeq 0.5724$ and that $g_{\theta}\left(\theta_{B}\right) g\left(\theta_{B}\right) \simeq 0.4392 \alpha^{2}$. Substitution of these values into (5.4) and (5.5) yields the formulae

$$
\begin{gathered}
x_{B} \simeq 2.1466\left(\gamma \alpha^{2}\right)^{-1}, \\
t_{B} \simeq 1.5179\left(\gamma \alpha^{2}\right)^{-1}+\varepsilon(0.2309) .
\end{gathered}
$$

The first of our numerical computations are displayed in Figs. 1 and 2 where we compare values of $x_{B}$ and $t_{B}$, respectively, derived using the exact methods of Sec. 3 with those obtained from the asymptotic methods of Sec. 4 . In both figures we take $\varepsilon=10^{-2}, \gamma=1$ and plot $x_{B}$ and $t_{B}$ against the amplitude parameter $\alpha$. The exact theory is represented by the solid line and the asymptotic theory is represented by the dashed line. It is apparent from both of these figures that the asymptotic method gives very good agreement with the exact solution and, in fact, for values of the amplitude parameter $\alpha$ above 6 the differences are in the third decimal place. What is not shown in these figures, however, is the fact that the computational time for the exact results is orders of magnitude greater than that for the asymptotic results. The overall trends in $x_{B}$ and $t_{B}$ as functions of $\alpha$ are clearly evident in these figures.

In Figs. 3 and 4 we plot $x_{B}$ and $t_{B}$, respectively, against $-\log _{10} \varepsilon$ over the range 0 to 4 for $\alpha=4$ and $\gamma=1$. This range of $-\log _{10} \varepsilon$ means that $\varepsilon$ varies from 1 to $10^{-4}$. In each figure the solid line represents the exact theory and the dashed line represents the result of the asymptotic analysis. The figures point out quite clearly that the approximate method gives excellent results in the range $\varepsilon \lesssim 10^{-1}$. Even for values of $\varepsilon$ which are larger the agreement between exact and approximate results are in qualitative agreement.

5.2. Example calculation with varying wall thickness. In this section we will examine the formation of a shock as predicted by the weakly nonlinear theory when the effects of a gradually varying tube wall thickness are included. As in the preceeding 


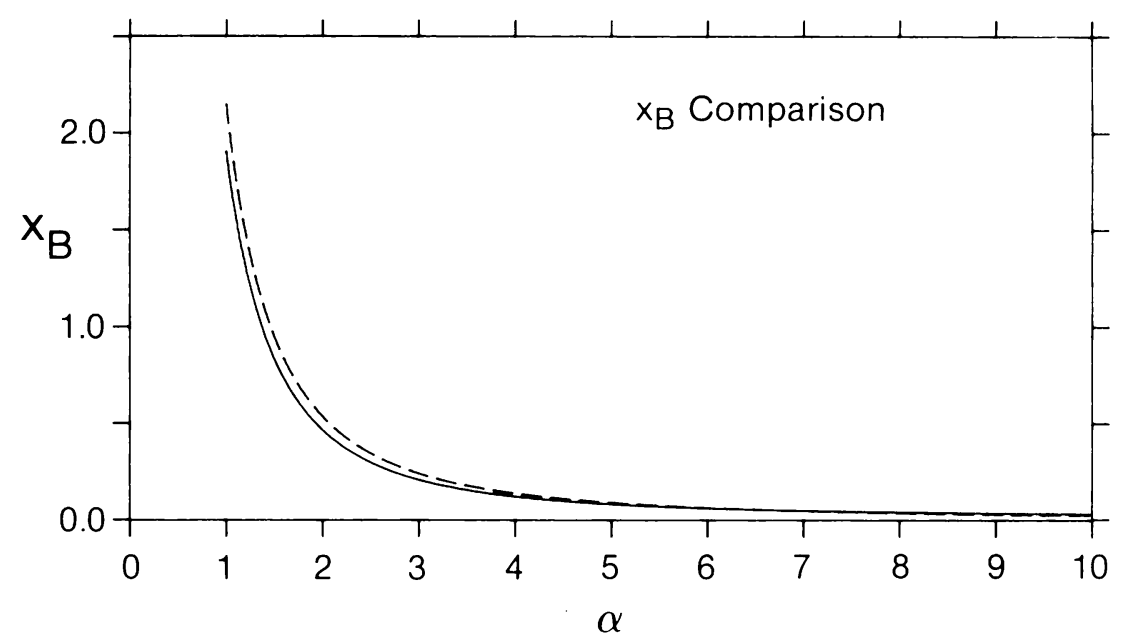

FiG. 1. Comparative plot of the position of first shock formation as calculated by the method of characteristics (solid line) and the asymptotic theory (dashed line) versus the boundary pressure amplitude parameter $\alpha$. The additional assumed parameter values are $\gamma=1.0$, $e=10^{-2}, A_{0}=1.0, e=0.0$, and $h_{0}=1.0$

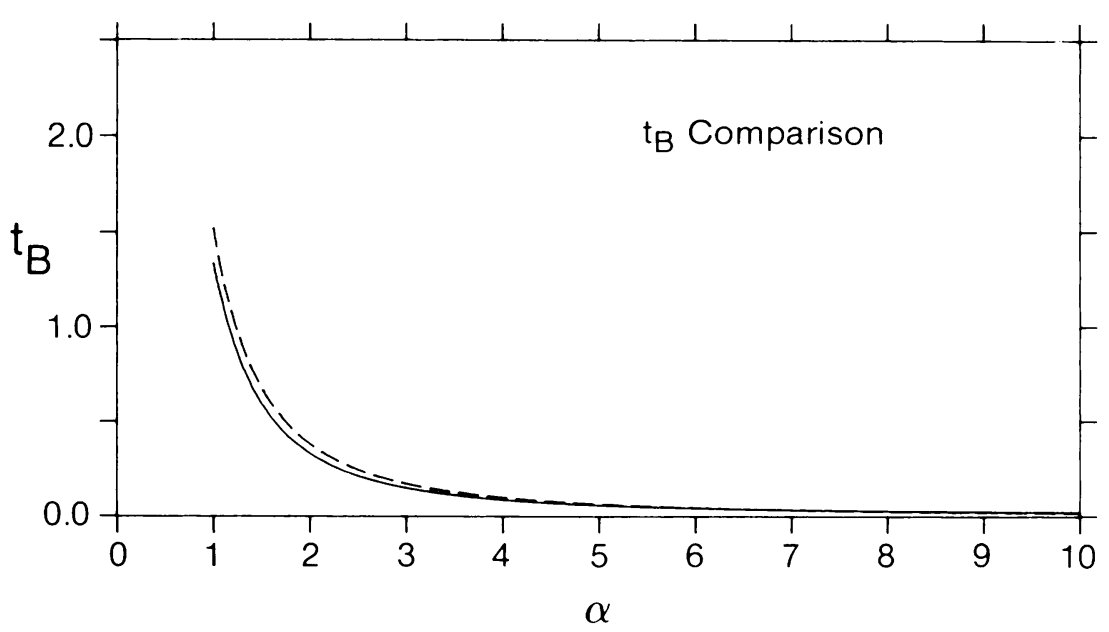

Fig. 2. Comparative plot of the time of first shock formation as calculated by the method of characteristics (solid line) and the asymptotic theory (dashed line) versus the boundary pressure amplitude parameter $\mathrm{r}$. The additional assumed parameter values are $;=1.0$. $\varepsilon=10^{-2}, A_{0}=1.0, e=0.0$, and $h+0=1.0$

subsection we will assume $A_{0} \equiv 1.0$ and $\varepsilon \equiv 0.0$. As well, we shall retain the Ishihara et. al strain energy model (5.2) and the applied boundary condition (5.1). However, in this section we will examine the consequences of two different models for the tube wall thickness corresponding to a thickening (or thinning) of limited axial extent, and another corresponding to a gradual transition to a thicker (or thinner) tube wall. 


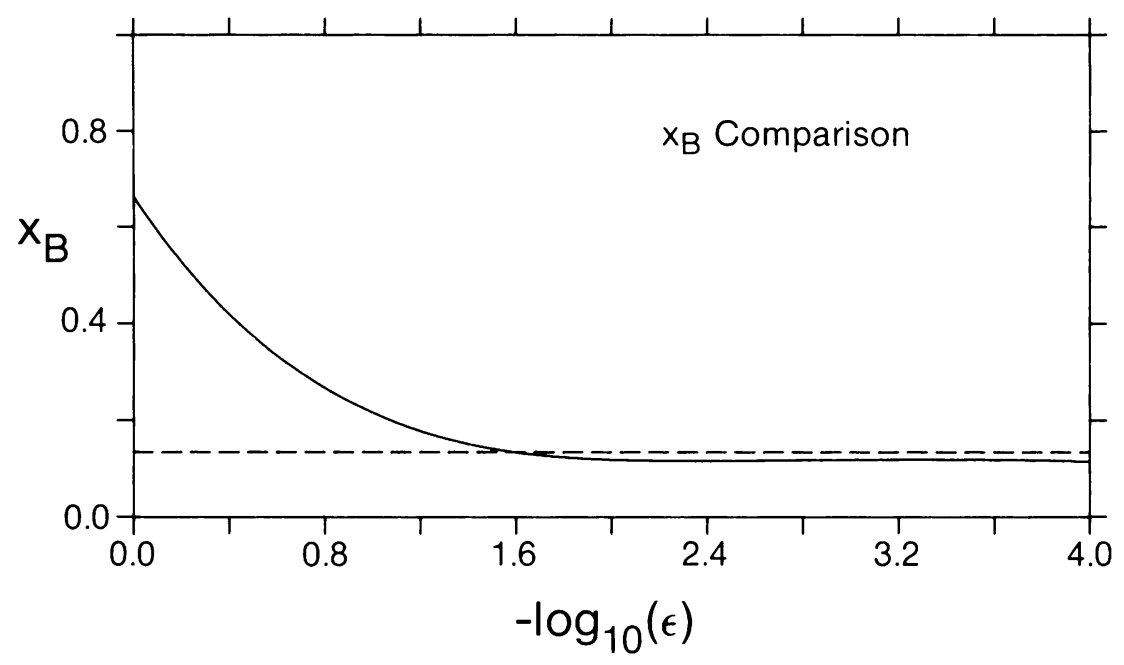

FIG. 3. Comparative plot of the position of first shock formation as calculated by the method of characteristics (solid line) and asymptotic theory (dashed line) versus the boundary pressure "period" parameter $\varepsilon$. The additional assumed parameter values are $\gamma=1.0, \alpha=4.0$, $e=0.0, A_{0}=1.0$, and $h_{0}=1.0$.

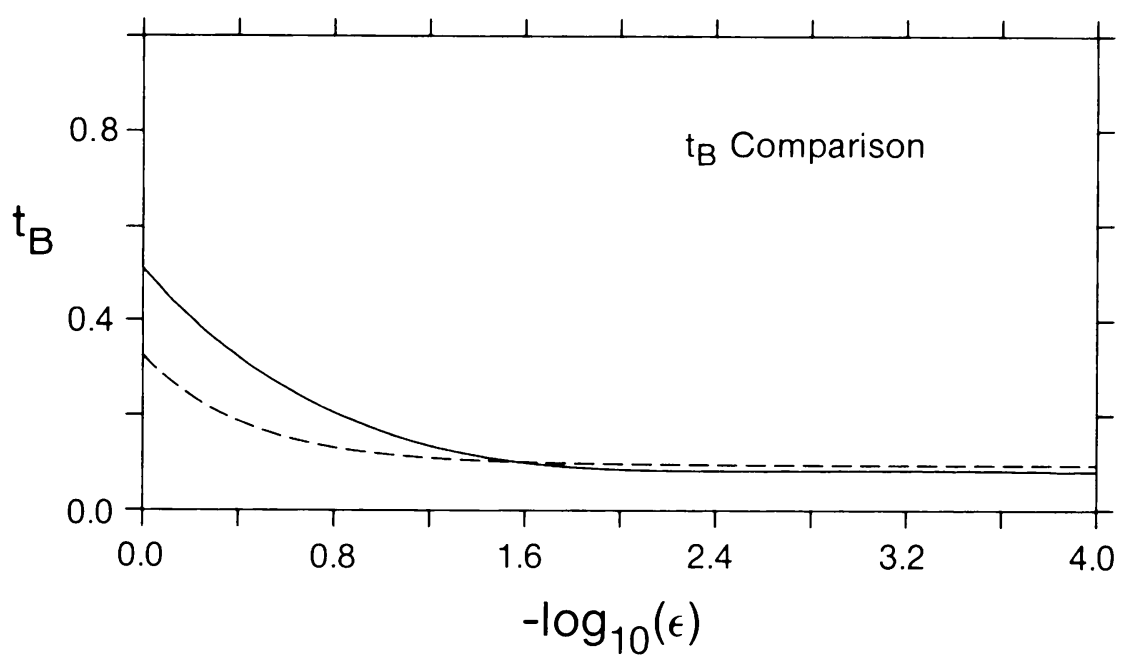

FIG. 4. Comparative plot of the time of first shock formation as calculated by the method of characteristics (solid line) and the asymptotic theory (dashed line) versus the boundary pressure "period" parameter $\varepsilon$. The additional assumed parameter values are $\gamma=1.0, \alpha=4.0$, $e=0.0, A_{0}=1.0$, and $h_{0}=1.0$ 
The two models are given, respectively, by

$$
\begin{gathered}
h_{0}(x)=1+\mu \exp \left[-\delta\left(x-x_{0}\right)^{2}\right], \\
h_{0}(x)=1+\mu\left\{1+\tanh \left[\delta\left(x-x_{0}\right)\right]\right\} / 2,
\end{gathered}
$$

where $\mu$ is an amplitude parameter, $x=x_{0}$ corresponds to the 'center' of the thickness feature, and $\delta$ will 'control' the axial extent of the transition. When $\mu>0(\mu<0)$ the above configurations will be said to correspond to a 'thickening' ('thinning') of the tube wall. The maximum absolute change in the thickness in either model in $|\mu|$.

From (4.3a) and (5.2) it follows that

$$
\varphi_{0}(x)=\left[2 h_{0}(x)\right]^{-1},
$$

and from (4.3d) and (5.2) it follows that $\beta \equiv 2 \gamma$. Consequently, from (4.24) the position of the shock will be given by

$$
\int_{0}^{x_{B}} h_{0}^{-2}(\eta) d \eta=\frac{2^{3 / 2}}{3}\left[\gamma g_{\theta}\left(\theta_{B}\right) g\left(\theta_{B}\right)\right]^{-1}
$$

where $\theta_{B}$ is as described in Sec. 5.1. This expression clearly shows that a thickening (thinning) of the tube wall will retard (accelerate) the formation of a shock.

It follows from (4.23) that the time of first shock formation will be given by

$$
t_{B}=2^{-1 / 2} \int_{0}^{x_{B}}\left[h_{0}(\eta)\right]^{-1 / 2} d \eta+\varepsilon\left\{\theta_{B}-\frac{3 \gamma}{4 \sqrt{2}} g^{2}\left(\theta_{B}\right) \int_{0}^{x_{B}}\left[h_{0}(\eta)\right]^{-2} d \eta\right\} \text {. }
$$

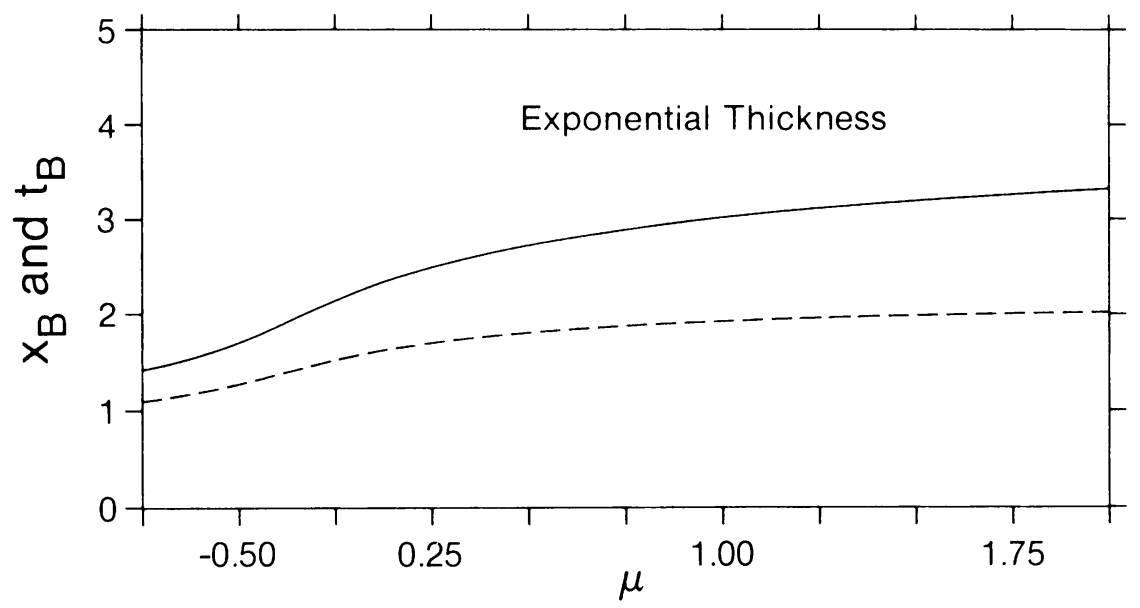

FIG. 5. Plot of the position (solid line) and time (dashed line) of first shock formation assuming the exponentially varying thickness model (5.7a) versus $\mu$ for the range $-0.5 \leq \mu \leq 2.0$ (with $\delta=4.0$ and $\left.x_{0}=1.5\right)$. The other parameter values are $\alpha=1.0, \varepsilon=10^{-1}$. $y=1.0, e=0.0$, and $x_{0}=1.0$. 


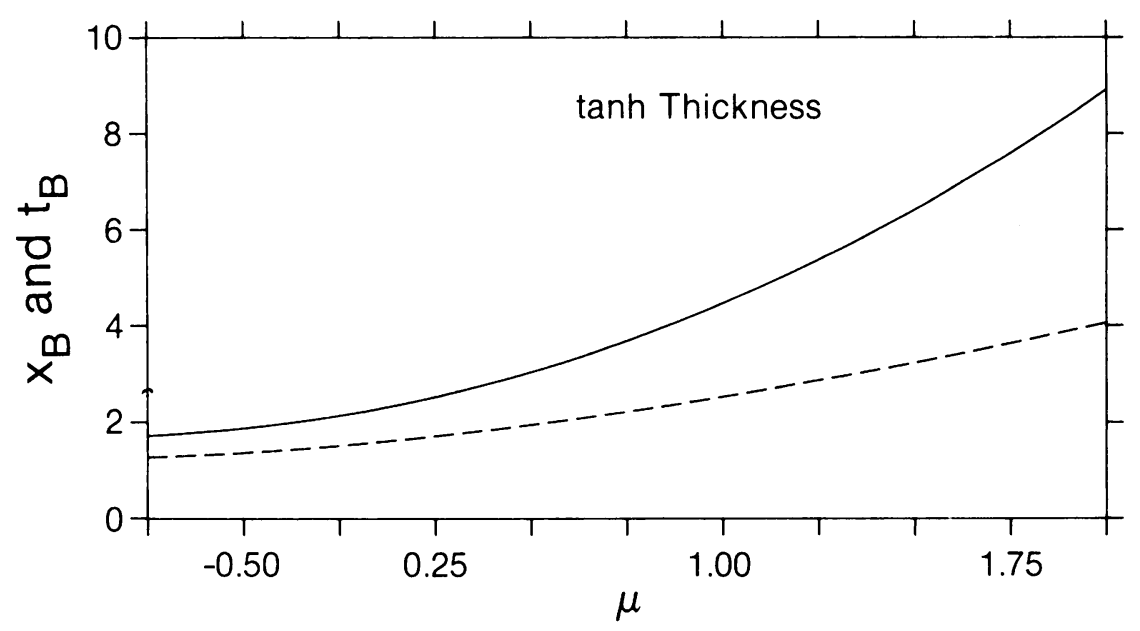

Fig. 6. Plot of the position (solid line) and time (dashed line) of first shock formation assuming the hyperbolic tangent thickness model (5.7b) versus $\mu$ for the range $-0.5 \leq \mu \leq 2.0$ (with $\delta=4.0$ and $x_{0}=1.5$ ). The other parameter values are $\alpha=1.0, \varepsilon=10^{-1}, \gamma=1.0$, $e=0.0$, and $A_{0}=1.0$.

Figures 5 and 6 present the results for $x_{B}$ and $t_{B}$ for the thickness models (5.7a) and $(5.7 \mathrm{~b})$ for $-0.5 \leq \mu \leq 2$, respectively, assuming the boundary pressure function (5.1) with $\alpha=1.0$ and $\varepsilon=10^{-1}$. In both figures the solid line corresponds to $x_{B}$ and the dashed line to $t_{B}$. As well, in both figures we have assumed $x_{0}=1.5$ and $\delta=4.0$. The strain energy function assumed was, again, the Ishihara model (5.2) with $\gamma=1.0$. Note that as the amplitude of the thickness anomaly (i.e., $\mu$ ) increases the position and time of first shock formation monotonically increases so that the shock initiates further down the tube.

6. Conclusion. We have modelled and analyzed an important class of nonlinear wave problems in liquid-filled distensible tubes and found, in contrast to earlier studies, that waves propagating into a region where the transmural pressure is zero will not break on the lead characteristic no matter what form the strain energy function assumes or the degree of axial strain to which the tube is subjected. Then with the ultimate goal of analyzing these problems in variable media we developed an asymptotic procedure based upon the theory of relatively undistorted waves. By comparing results derived using this method with those based upon an exact procedure for the restricted case of a homogeneous medium we found the asymptotic method gave very good agreement and, in particular, this agreement improved with increasing values of the pulse amplitude parameter. Having 'established' the validity of the approximate procedure we then employed it to investigate the influence of wall thickness changes upon shock initiation times and distances. We found that wall thickening retards shock formation whereas thinning has the opposite effect.

The development of a rational theory based on nonlinear elasticity shows that the pressure-area relation must be of a particular form (i.e., (2.16)). This form precludes 
the formation of shocks on the lead characteristic. This means that if one were to employ, for example, the theory of acceleration front analysis to determine whether or not shocks form, one would be led to the incorrect conclusion that they did not.

Acknowledgment. The authors would like to thank Professor Robert J. Tait for periodic discussions on this work. Preparation of this manuscript was supported in part by Operating Research Grants (A4039 to T.B.M. and A9357 to G.E.S.) awarded to the authors by the Natural Sciences and Engineering Research Council of Canada.

\section{REFERENCES}

[1] G. Rudinger, Shock waves in mathematical models of the aorta, J. Appl. Mech. 37, 34-37 (1970)

[2] J. W. Lambert, Fluid Flow in a Nonrigid Tube, Doctoral Dissertation Series 19, 418 pp., University Microfilms Inc., Ann Arbor, Mich., 1956

[3] T. B. Moodie and J. B. Haddow, Waves in thin-walled elastic tubes containing an incompressible inviscid fluid, Internat. J. Non-Linear Mech. 12, 223-231 (1977)

[4] J. R. Womersley, An elastic tube theory of pulse transmission and oscillatory flow in mammalian arteries, Wright Air Development Center Technical Report TR56-614 (1957)

[5] R. Skalak, Wave propagation in blood flow, Biomechanics Symposium (Ed. Y. C. Fund), New York, A.S.M.E.

[6] P. J. Chen and M. F. McCarthy, Unsteady flow of incompressible fluids in deformable tubes, Acta Mech. 33, 189-197 (1979)

[7] M. Anliker, R. L. Rockwell, and E. Ogden, Non-linear analysis of flow pulses and shock waves in arteries, Parts I and II, Z. Angew. Math. Phys. 22, 217-246 and 563-581 (1971)

[8] S. J. Cowley, Elastic jumps on fluid-filled elastic tubes, J. Fluid Mech. 116, 459-473 (1982)

[9] S. J. Cowley, On the wavetrains associated with elastic jumps on fluid-filled elastic tubes, Quart. J. Mech. Appl. Math. 36, 289-312 (1983)

[10] R. J. Tait and T. B. Moodie, Waves in nonlinear fluid filled tubes, Wave Motion 6, 197-203 (1984)

[11] E. Varley and E. Cumberbatch, Non-linear, high frequency sound waves, J. Inst. Maths. Applics. 2, 133-143 (1966)

[12] B. R. Seymour and M. P. Mortell, Nonlinear geometrical acoustics, Mechanics Today, Vol. 2 (edited by S. Nemat-Nasser), Pergamon, New York, 1975, pp. 251-312

[13] R. W. Ogden, Non-Linear Elastic Deformations, Ellis Horwood, Chichester, 1984

[14] A. J. Chorin and J. E. Marsden, A Mathematical Introduction to Fluid Mechanics, Springer Verlag, New York, 1979

[15] G. B. Whitham, The propagation of weak spherical shocks in stars, Comm. Pure Appl. Math. 6, 197-414 (1953)

[16] G. B. Whitham, On the propagation of weak shock waves, J. Fluid Mech. 1, 290-318 (1956)

[17] A. Ishihara, N. Hashitsume, and M. Tabibana, Statistical theory of rubber-like elasticity, iv. Twodimensional stretching, J. Chem. Phys. 19, 1508-1511 (1951)

[18] G. E. Swaters, Resonant three-wave interactions in nonlinear hyperelastic fluid-filled tubes, J. Appl. Math. Physics (ZAMP), 39, 668-681 (1988) 